\title{
Heating-Induced Long-Range $\eta$ Pairing in the Hubbard Model
}

\author{
J. Tindall, ${ }^{1, *}$ B. Buča,${ }^{1}$ J. R. Coulthard, ${ }^{1}$ and D. Jaksch ${ }^{1,2}$ \\ ${ }^{1}$ Clarendon Laboratory, University of Oxford, Parks Road, Oxford OX1 3PU, United Kingdom \\ ${ }^{2}$ Centre for Quantum Technologies, National University of Singapore, 3 Science Drive 2, Singapore 117543
}

(Received 13 February 2019; published 17 July 2019)

\begin{abstract}
We show how, upon heating the spin degrees of freedom of the Hubbard model to infinite temperature, the symmetries of the system allow the creation of steady states with long-range correlations between $\eta$ pairs. We induce this heating with either dissipation or periodic driving and evolve the system towards a nonequilibrium steady state, a process which melts all spin order in the system. The steady state is identical in both cases and displays distance-invariant off-diagonal $\eta$ correlations. These correlations were first recognized in the superconducting eigenstates described in Yang's seminal Letter [Phys. Rev. Lett. 63, 2144 (1989)], which are a subset of our steady states. We show that our results are a consequence of symmetry properties and entirely independent of the microscopic details of the model and the heating mechanism.
\end{abstract}

DOI: 10.1103/PhysRevLett.123.030603

Introduction.-Driving and dissipation have recently emerged as transformative tools for dynamically evolving quantum systems into nonequilibrium phases with desirable properties [1-3]. In the context of strongly correlated many-body systems, these tools can drastically alter their microscopic behavior and manifest a variety of collective and cooperative phenomena at the macroscopic level.

Controlled dissipation, for example, has been shown to be a versatile resource for quantum information and simulation purposes $[4,5]$. Recent proposals show how, in ultracold atomic systems, Markovian baths which act quasilocally can drive the system towards pure steady states with exotic properties such as superfluidity [6,7]. These schemes, however, rely on precise engineering of the Lindblad jump operators in order to target specific states and avoid the system heating up to a generic thermal ensemble.

Meanwhile, significant interest has been generated by the superconducting-like states that have been induced in a variety of materials by transient excitation of the vibrational degrees of freedom (d.o.f.) using terahertz laser pulses $[8,9]$. These optically driven systems are often modeled via Floquet driving - where the Hamiltonian is subject to a time-dependent periodic field [10-12]. Through careful choice of the driving parameters the effective Hamiltonian can be modified, on comparatively short timescales, to one which favors superconductivity, and so the system may

Published by the American Physical Society under the terms of the Creative Commons Attribution 4.0 International license. Further distribution of this work must maintain attribution to the author(s) and the published article's title, journal citation, and DOI. transiently reach a superconducting prethermal state. However, Floquet heating [13-15] means that in most cases these systems continue to absorb energy from the driving field, heating them up and eventually destroying any superconducting order present.

In this Letter we show that, counterintuitively, the interplay between symmetry and heating can actually lead to the formation of steady states with coherent, long-range correlations. This heating can be achieved either with dephasing or with periodic driving and, as the generation of these correlations is based on symmetry, the microscopic details of the heating mechanism are unimportant. We believe this could mark an important step in explaining why such states are being observed in experiments $[8,9]$ where the stringent conditions required in previous numerical work $[10-12,16]$ are not fulfilled.

In the aforementioned steady states we demonstrate how the formation of these long-range correlations results from the melting of all order in the complementary symmetry sector where the heating is applied. The competition between different types of order is a mechanism considered to underpin the formation of transient superconductivity $[17,18]$ and thus we focus our work within this context.

Specifically, we apply driving or dephasing induced heating to the spin d.o.f. of bipartite $D$-dimensional realizations of the Hubbard model, melting any order in this sector, and reaching robust mixed states with completely uniform long-range correlations between $\eta$ pairs. This pairing is known to provide a possible mechanism for superconductivity [18-21]. Compared to previous proposals, which excite $\eta$-paired states through carefully tuned driving or dissipation $[6,16]$, our results are based on symmetry arguments. This means that the engineered steady states are independent of the model parameters 
and, for arbitrary initial states, guaranteed to have completely uniform $\eta$ correlations. In the case of dephasing we prove the results of our simulations by block diagonalizing the Liouvillian superoperator, giving an explicit parametric form for the steady states.

Model.-The Hubbard model, in second quantized form, reads

$$
H=-\tau \sum_{\langle i j\rangle, \sigma}\left(c_{\sigma, i}^{\dagger} c_{\sigma, j}+\text { H.c. }\right)+U \sum_{i} n_{\uparrow, i} n_{\downarrow, i},
$$

where $c_{\sigma, i}^{\dagger}$ and its adjoint are the usual creation and annihilation operators for a fermion of spin $\sigma \in\{\uparrow, \downarrow\}$ on site $i$. Additionally, $n_{\sigma, i}$ is the number operator for a particle of spin $\sigma$ on site $i$, and $\tau$ and $U$ play the role of kinetic and interaction energy scales, respectively. In this work, we set $\hbar=1$.

The Hamiltonian in Eq. (1) has a rich structure due to the two $\mathrm{SU}(2)$ symmetries it possesses [22]. The first of these is the "spin" symmetry, which relates to spinful particles (singlons) $\sigma \in\{\uparrow, \downarrow\}$. The second, often referred to as " $\eta$ symmetry," is central to this Letter and relates to spinless quasiparticles (doublons and holons) $\sigma \in\{\uparrow \downarrow$, vac $\}$. It can be interpreted as a type of particle-hole symmetry. This $\eta$ symmetry is revealed by introducing the associated operators,

$$
\begin{aligned}
\eta^{+} & =\sum_{i} \eta_{i}^{+}=\sum_{i}(-1)^{i} c_{\uparrow, i}^{\dagger} c_{\downarrow, i}^{\dagger}, \\
\eta^{-} & =\sum_{i} \eta_{i}^{-}=\sum_{i}(-1)^{i} c_{\downarrow, i} c_{\uparrow, i}, \\
\eta^{z} & =\sum_{i} \eta_{i}^{z}=\sum_{i} \frac{1}{2}\left(n_{i, \uparrow}+n_{i, \downarrow}-1\right),
\end{aligned}
$$

with $\eta_{i}^{+}\left(\eta_{i}^{-}\right)$describing the creation (annihilation) of a doublon on site $i$ with an alternating site-dependent phase. The operators in Eq. (2) fulfill the relations $\left[H, \eta^{ \pm}\right]=0$ and $\left[H, \eta^{+} \eta^{-}\right]=\left[H, \eta^{z}\right]=0$ and commute with all the generators of the spin symmetry.

The presence of $\eta$-pairing superconductivity in the Hubbard model is a phenomenon first recognized by Yang in his seminal Letter [23]. There it was proved that the pure states $|\psi\rangle \propto\left(\eta^{+}\right)^{N}|\mathrm{vac}\rangle$ are eigenstates of $H$ and possess off-diagonal long-range order (ODLRO) in the form of doublon-doublon correlations,

$$
\operatorname{Tr}\left(\rho \eta_{i}^{+} \eta_{j}^{-}\right)=\mathrm{const} \quad \forall i, j, \quad i \neq j,
$$

where $\rho=|\psi\rangle\langle\psi|$. This relation provides a possible definition of superconductivity, as a finite value of this quantity can be shown to imply both the Meissner effect and flux quantization $[19,24,25]$. These states, however, are excited states of $H$, and the long-range order they possess is not usually seen in physical states (ground states, thermal states, etc.) of the model due to destructive interference from the short-range coherences involving spinful particles. By driving the Hubbard model in the spin basis these are destroyed and we can consistently engineer states with long-range uniform correlations in $\left\langle\eta_{i}^{+} \eta_{j}^{-}\right\rangle$.

As our primary mechanism for achieving this we consider the Hubbard model immersed in an environment which induces local dephasing in the spin basis. This model is motivated by the spin fluctuation theory of superconductivity, where electrons pair due to their scattering on spin fluctuations [26-28]. Our dephasing mimics these scattering events.

Despite the "toy" nature of our model, the Hubbard Hamiltonian can be accurately realized by loading ultracold fermionic atoms into optical lattices [29-31]. These quantum simulators offer precise experimental control over the microscopic details of the system. In the Supplemental Material (SM) [32] we show how dephasing can occur by immersing the lattice into a homogeneous Bose-Einstein condensate $[40,41]$. If the interactions are tuned with Feshbach resonances $[42,43]$ so that the scattering amplitudes between the two fermionic spin states and the bosons are equal in magnitude and opposite in sign, then dephasing will occur solely in the spin sector.

Results.-We couple the Hubbard Hamiltonian in Eq. (1) to an environment which applies spin dephasing on each site of an $M$-site lattice. The ensuing dynamics is modeled, under the Markov approximation, via the Lindblad master equation,

$\frac{\partial \rho}{\partial t}=\mathcal{L} \rho=-i[H, \rho]+\gamma \sum_{j}\left(L_{j} \rho L_{j}^{\dagger}-\frac{1}{2}\left\{L_{j}^{\dagger} L_{j}, \rho\right\}\right)$,

$L_{j}=s_{j}^{z}=n_{\uparrow, j}-n_{\downarrow, j}$,

with Lindblad operators $s_{j}^{z}$ on each site $j$. Because these operators are restricted to the spin $\mathrm{SU}(2)$ symmetry sector, we obtain $\left[L_{j}, \eta^{ \pm}\right]=\left[L_{j}, \eta^{z}\right]=0 \forall j$.

For Eq. (4), any operator $A$ which satisfies

$$
[H, A]=\left[L_{j}, A\right]=\left[L_{j}^{\dagger}, A\right]=0 \quad \forall j
$$

is a null eigenvector of $\mathcal{L}$ and the adjoint map $\mathcal{L}^{\dagger}$, which means the associated observable $\langle A\rangle$ is conserved. Thus, the map in Eq. (4) conserves $\left\langle\eta^{+} \eta^{-}\right\rangle,\left\langle\eta^{z}\right\rangle$, and $\left\langle S^{z}\right\rangle=\sum_{i}\left\langle s_{i}^{z}\right\rangle$. Through a power series expansion, any appropriately trace normalized function $f\left(\eta^{+} \eta^{-}, \eta^{z}, S^{z}\right)$ of these operators is a steady state of $\mathcal{L}$; i.e., if $\rho_{s s}=f\left(\eta^{+} \eta^{-}, \eta^{z}, S^{z}\right)$, then $\mathcal{L} \rho_{s s}=0$. In the SM [32] we show that steady states of this form have $\operatorname{Tr}\left(\rho_{s s} \eta_{i}^{+} \eta_{j}^{-}\right)=$ const $\forall i, j$ (see also Ref. [44]). We also show how this result of heating-induced long-range order can be extended to arbitrary models with multiple $\mathrm{SU}(2)$ symmetries. We emphasize that the expected steady state $\rho_{s s}$ is realizable for 
any bipartite $D$-dimensional realization of the spindephased Hubbard model.

Here, we focus on 1D lattice realizations due to their numerical tractability. We perform calculations where the system is initialized in a given state and then evolved by solving the time-dependent master equation in Eq. (4) and quenching $U$, providing a direct comparison with the dynamics of the closed system. In the open system the value of $U$ which we quench to solely sets the timescale of relaxation and long-range order will always appear. We use a quantum trajectories approach [45] to solving Eq. (4) combined with density matrix renormalization group [46] and time evolving block decimation [47] algorithms for finding the ground state and evolving the wave function of the system, respectively. These simulations were performed with the Tensor Network Theory library [48]. Further numerical details and analysis can be found in the SM [32].

In Fig. 1. we plot, for various distances $j$, the averaged quantity $\left\langle\eta_{i}^{+} \eta_{i+j}^{-}\right\rangle$over time for a quench from the ground state of the Hubbard Hamiltonian. We also include the matrix of correlations of $\left\langle\eta_{i}^{+} \eta_{j}^{-}\right\rangle$for the density matrix following the quench. For comparison, we show the case where the system is not coupled to the environment, $\gamma=0$. When the environment is present there is a relaxation of the system to a steady state which possesses the order (a)

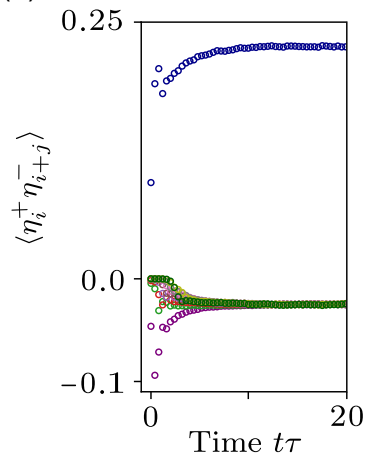

(c)

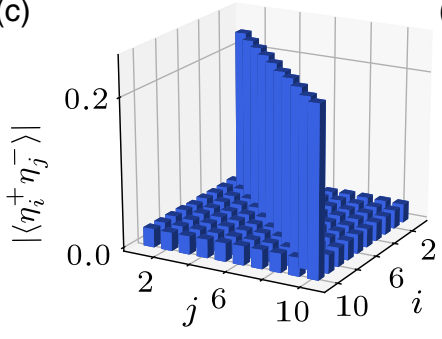

(b)

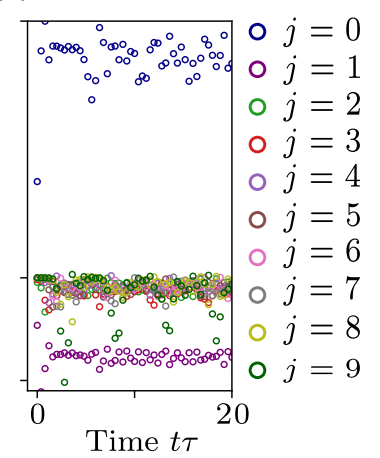

(d)

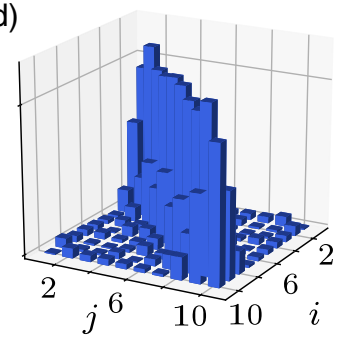

FIG. 1. (a),(b) Evolution of the correlator $\left\langle\eta_{i}^{+} \eta_{i+j}^{-}\right\rangle$, averaged over all sites separated by a distance $j$, for the 10 site Hubbard model at half filling and $\left\langle S^{z}\right\rangle=0$. The system is initialized in the ground state of $H$ for $U=4.0 \tau$ and then evolved with a systemenvironment coupling of $\gamma=2.0 \tau$ for (a) and $\gamma=0.0$ for (b). In both quenches the interaction strength is changed to $U=\tau$. (c), (d) Matrix of correlations of $\left|\left\langle\eta_{i}^{+} \eta_{j}^{-}\right\rangle\right|$associated with the density matrix at $t \tau=20.0$ for the simulations in (a) and (b), respectively. expressed in Eq. (3); the value of $\left\langle\eta_{i}^{+} \eta_{j}^{-}\right\rangle$is finite and constant for all $i \neq j$. We observe that this is facilitated by a decrease in the short-range $\eta$-pairing correlations in order to allow the long-range ones to increase: the nonequilibrium dynamics involve a "spreading" of the $\eta$ correlations over all length scales of the system, which is necessary due to the conservation of $\eta^{+} \eta^{-}$. Plots of the corresponding doublon momentum distribution are discussed in the SM [32].

We also show how this ODLRO is observable even under perturbative and unwanted dephasing in the model. We do this by introducing "charge dephasing" using jump operators that do not solely act in the spin basis, breaking the strong symmetry relations and causing the steady state of the Liouvillian to contain no coherences. Nonetheless, we set the strength of the charge dephasing to values around $1 \%$ of the spin dephasing and find there exists an intermediate timescale where the results of Fig. 1 can be observed. We also find that the window in which the uniform long-range correlations exist can be directly controlled by the ratio of the couplings for the spin dephasing and the charge dephasing: the dissipative evolution induced by the charge dephasing becomes increasingly frozen out as this ratio increases. In particular, our data suggest that the length of this window can be extended by increasing the coupling of the spin dephasing while the other parameters in the model remain constant.

To obtain a more intuitive idea of the nature of the steady state, $\rho_{s s}=f\left(\eta^{+} \eta^{-}, \eta^{z}, S^{z}\right)$, we consider a physically motivated parametrization of the steady state function,

$$
\rho_{s s} \propto \exp \left(\mu_{1} \eta^{+} \eta^{-}+\mu_{2} N_{\uparrow}+\mu_{3} N_{\downarrow}\right),
$$

where we have exchanged $\eta^{z}$ and $S^{z}$ with $N_{\uparrow}=\sum_{i} n_{\uparrow, i}$ and $N_{\downarrow}=\sum_{i} n_{\downarrow, i}$, as they can be expressed as linear combinations of these operators. Calculations on small lattices show that the parametrization in Eq. (6) captures all relevant observables when compared to numerical simulations which reach the long-time limit in Eq. (4). Equation (6) describes a generalized grand-canonical-like equilibrium (GCE) state with the Lagrange multipliers $\mu_{1}$, $\mu_{2}, \mu_{3}$ associated to each of the conserved quantities. Notably, the Lagrange multiplier for the Hamiltonian is 0 , i.e., at infinite temperature. As is typical of states in this form, the Lagrange multipliers are independent of the quench parameters and are governed solely by the values of the conserved quantities $\left\langle\eta^{+} \eta^{-}\right\rangle,\left\langle N_{\uparrow}\right\rangle$, and $\left\langle N_{\downarrow}\right\rangle$ associated with the initial state. The existence of a "quadratic" charge $\left(\eta^{+} \eta^{-}\right)$in the GCE state is crucial for the presence of nontrivial long-range order and sets it apart from generalized Gibbs ensemble states [49-52].

To emphasize the properties of the stationary state in Eq. (6), we plot, in Fig. 2., the projection of the density matrix in the spin $(s)$ and $\eta(d)$ d.o.f. $\mathcal{P}_{s, d} \rho \mathcal{P}_{s, d}$. Explicitly, these projectors remove any basis vectors which contain, 


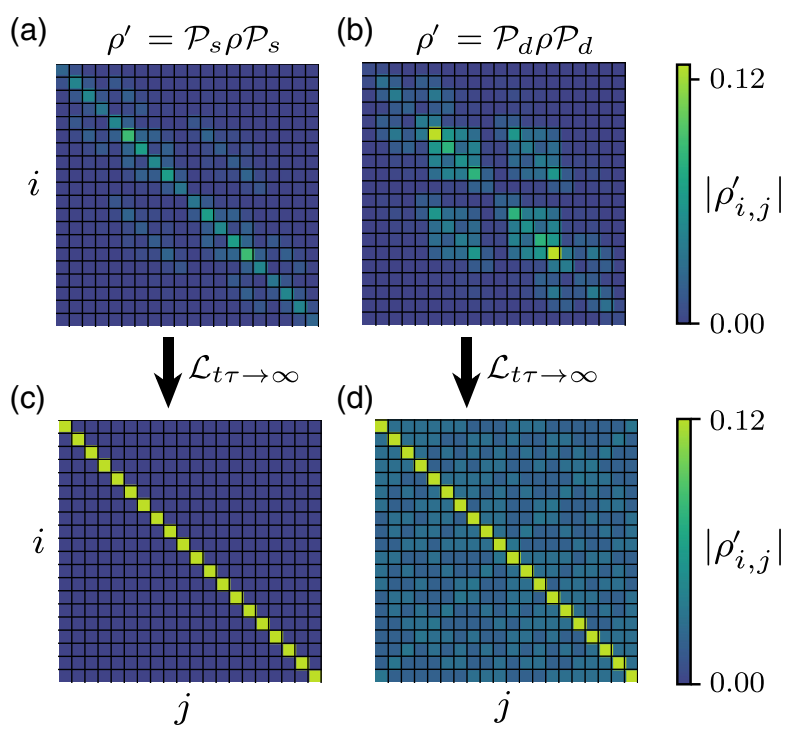

FIG. 2. Projections, $\rho^{\prime}=\mathcal{P}_{s, d} \rho \mathcal{P}_{s, d}$, of the density matrix in the spin $(s)$ and $\eta(d)$ sectors of the $M=6$ Hubbard model. The system is at symmetric half filling, $\left\langle N_{\uparrow}\right\rangle=\left\langle N_{\downarrow}\right\rangle=M / 2$, and the projections have been renormalized $\left[\operatorname{Tr}\left(\rho^{\prime}\right)=1\right]$. The color indicates the magnitude of the matrix elements.. Following the projection, the indices $i$ and $j$ run over the remaining basis states in lexicographic order when they are converted to binary strings, where $\uparrow=1, \downarrow=0$ and $\uparrow \downarrow=1$, vac $=0$ for the projections in $s$ and $d$, respectively. As an example, for plots (a) and (c), when $i=1$, this corresponds to the basis vector $|\uparrow \uparrow \uparrow \downarrow \downarrow \downarrow\rangle=|111000\rangle$, and when $i=20$, $|\downarrow \downarrow \downarrow \uparrow \uparrow \uparrow\rangle=|000111\rangle$. (a),(b) Projections for the thermal state of the $U=2.5 \tau$ Hubbard model: $\rho \propto \exp (-\beta H)$ with $\beta=0.8 / \tau$. (c),(d) Projections following spin dephasing of this initial state by the map in Eq. (4) in the long-time limit with quench parameters $U=\tau$ and $\gamma=2.0 \tau$.

respectively, doublons $(\sigma \in\{\uparrow \downarrow, v a c\})$ and singlons $(\sigma \in\{\uparrow, \downarrow\})$ on any lattice sites. We plot these projections following a quench from a thermal state of the Hubbard model. Initially [Figs. 2(a) and 2(b)], the system contains coherences in both these sectors which decay with distance - additionally (not pictured), the density matrix contains coherences between doublons and singlons. Following spin dephasing in the long-time limit, any coherences involving singlons are destroyed resulting in an infinite temperature ensemble in the spin symmetry sector [Fig. 2(c)]. Only coherences involving doublons and holons remain [Fig. 2(d)]. These are completely distance invariant, as described by the parametrization in Eq. (6).

The strong symmetries [53] $\left\langle\eta^{+} \eta^{-}\right\rangle,\left\langle N_{\uparrow}\right\rangle$, and $\left\langle N_{\downarrow}\right\rangle$ can be used to block diagonalize the Liouvillian and, hence, the degeneracy of $\rho_{s s}$ is determined by the distinct combinations of the eigenvalues of these operators. The steady states are the states with maximum $\eta$ order in each block, quantified by the amplitude of the uniform off-diagonal correlations $\left|\left\langle\eta_{i}^{+} \eta_{j}^{-}\right\rangle\right|, i \neq j$. Notably, Yang's states $|\psi\rangle \propto$ $\left(\eta^{+}\right)^{N} \mid$ vac $\rangle$ form a subset of these steady states. They are the only pure steady states as they exist in the decoherence free subspace [54] of Eq. (4).

The infinite temperature ensemble pictured in Fig. 2(c) shows that the dephasing has continuously pumped energy into the spin d.o.f. of the lattice until it saturates and reaches infinite temperature. An alternative method of achieving this is through Floquet heating. To show this, we consider the closed Hubbard model under periodic driving in the form of a time-dependent inhomogeneous magnetic field:

$$
H(t)=H+B(t) \sum_{i=1}^{L} f(i) s_{i}^{z}
$$

with $B(t)=V \cos (\Omega t)$ and $f(i)$ describing the inhomogeneity. In the long-time limit the driving is expected to thermalize the system to infinite temperature $[13,14]$. However, because in this case the driving term commutes with all the generators of the $\eta$ symmetry, we expect heating to occur only within the spin sector.

In Fig. 3 we show how, by time evolving an initial state under $H(t)$, we realize long-time dynamics identical to that induced by the spin dephasing. By resonantly driving the
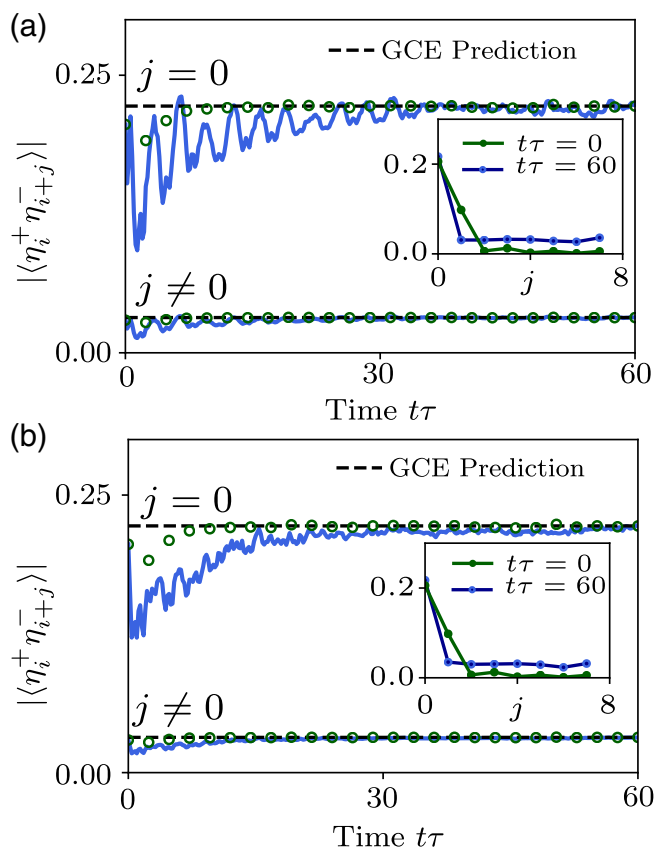

FIG. 3. Dynamics of the averaged $\eta$ correlations $\left|\left\langle\eta_{i}^{+} \eta_{i+j}^{-}\right\rangle\right|$ following a quench from the $U=\tau$ ground state of the $M=8$ half-filled Hubbard model with $\left\langle S^{z}\right\rangle=0$. The blue curves reflect a quench with the periodically driven Hamiltonian in Eq. (7), where $U=6.0 \tau$ and $\Omega=\tau$. The green markers are for a quench under the dephasing map in Eq. (4) with $U=\tau$ and $\gamma=2.0 \tau$. The dashed lines indicate the prediction from the grand-canonical ensemble in Eq. (6). Insets show the dependence of the correlations on distance, extracted for the blue curve at times $t \tau=0.0$ and $t \tau=60.0$. (a) $V=2.0 U$ and $f(i)=i$. (b) $V=4.0 U$ and $f(i) \in \operatorname{rand}[0,2.0]$, a uniform random number on the specified interval. 
system, $U=n \Omega n \in \mathbb{Z}^{+}$, the system thermalizes quickly [15] and the observables are in good agreement with both the grand-canonical description of Eq. (6) and long-time simulations of the map in Eq. (4). There is completely uniform off-diagonal long-range order in $\eta$ pairs (Fig. 3, insets).

In Fig. 3(a) we choose a linear magnetic field $f(i)=i$, while in Fig. 3(b) we choose a disordered field. In both cases the long-time dynamics are the same and this emphasizes that the choice of driving parameters and inhomogeneity is arbitrary - they only affect the timescale on which the system relaxes.

Conclusion.-We have demonstrated that long-range $\eta$ pairing can be created and protected within the Hubbard model by directly heating the spinful d.o.f. to infinite temperature. This destroys any coherences involving spinful particles which, in turn, creates uniform long-range correlations between the $\eta$ quasiparticles in the lattice.

Recent work has demonstrated that, with a judicious choice of parameters, applying a Gaussian pulse of a specific duration to the Mott-insulating phase of the Hubbard model can excite $\eta$ pairs [16]. This driving does not commute with the generators of the $\eta$ symmetry and, hence, uniform long-range correlations are not guaranteed. This does, however, open up the possibility of applying our heating scheme in conjunction with a similar driving process which can enhance the $\eta$ correlations in the system. Our heating would then continually spread these over all length scales, creating a long-lived superconducting state.

We also anticipate further work using an alternate heating mechanism within the Hubbard model, or $t-J$ model, which protects singlets and destroys other coherences. This could enhance superexchange pairing and induce superconductivity through nearest-neighbor singlet pairing $[10,55,56]$.

Finally, we emphasize that the results of this work are due to the multiple SU(2) symmetries of the Hubbard model and not its microscopic details. Hence, ODLRO can be realized through dephasing in any model with multiple symmetries (see SM [32]); as examples, we suggest multiband Hubbard models and the Richardson-Gaudin model [57], which also permit superconducting regimes.

We would like to acknowledge S.R. Clark for our fruitful conversations and his beneficial input on optical modulation. We also thank C. Sánchez Muñoz, J. Mur-Petit, H. Gao, and J. De Nardis for useful discussions. This work has been supported by EPSRC Grants No. EP/P009565/1 and No. EP/K038311/1 and is partially funded by the European Research Council under the European Union's Seventh Framework Programme (FP7/ 2007-2013)/ERC Grant Agreement No. 319286 Q-MAC. We acknowledge the use of the University of Oxford Advanced Research Computing (ARC) facility in carrying out this work. joseph.tindall@physics.ox.ac.uk

[1] F. Verstraete, M. M. Wolf, and C. J. Ignacio, Quantum computation and quantum-state engineering driven by dissipation, Nat. Phys. 5, 633 (2009).

[2] A. Cavalleri, Photo-induced superconductivity, Contemp. Phys. 59, 31 (2018).

[3] J. Eisert, M. Friesdorf, and C. Gogolin, Quantum manybody systems out of equilibrium, Nat. Phys. 11, 124 (2015).

[4] S. Touzard, A. Grimm, Z. Leghtas, S. O. Mundhada, P. Reinhold, C. Axline, M. Reagor, K. Chou, J. Blumoff, K. M. Sliwa, S. Shankar, L. Frunzio, R. J. Schoelkopf, M. Mirrahimi, and M. H. Devoret, Coherent Oscillations Inside a Quantum Manifold Stabilized by Dissipation, Phys. Rev. X 8, 021005 (2018).

[5] H. P. Lüschen, P. Bordia, S. S. Hodgman, M. Schreiber, S. Sarkar, A. J. Daley, M. H. Fischer, E. Altman, I. Bloch, and U. Schneider, Signatures of Many-Body Localization in a Controlled Open Quantum System, Phys. Rev. X 7, 011034 (2017).

[6] S. Diehl, A. Micheli, A. Kantian, B. Kraus, H. P. Büchler, and P. Zoller, Quantum states and phases in driven open quantum systems with cold atoms, Nat. Phys. 4, 878 (2008).

[7] B. Kraus, H. P. Büchler, S. Diehl, A. Kantian, A. Micheli, and P. Zoller, Preparation of entangled states by quantum Markov processes, Phys. Rev. A 78, 042307 (2008).

[8] W. Hu, S. Kaiser, D. Nicoletti, C. R. Hunt, I. Gierz, M. C. Hoffmann, M. Le Tacon, T. Loew, B. Keimer, and A. Cavalleri, Optically enhanced coherent transport in $\mathrm{YBa}_{2} \mathrm{Cu}_{3} \mathrm{O}_{6.5}$ by ultrafast redistribution of interlayer coupling, Nat. Mater. 13, 705 (2014), article.

[9] M. Mitrano, A. Cantaluppi, D. Nicoletti, S. Kaiser, A. Perucchi, S. Lupi, P. Di Pietro, D. Pontiroli, M. Riccò, S. R. Clark, D. Jaksch, and A. Cavalleri, Possible light-induced superconductivity in $\mathrm{K}_{3} \mathrm{C}_{60}$ at high temperature, Nature (London) 530, 461 (2016).

[10] J. R. Coulthard, S. R. Clark, S. Al-Assam, A. Cavalleri, and D. Jaksch, Enhancement of superexchange pairing in the periodically driven Hubbard model, Phys. Rev. B 96, 085104 (2017).

[11] M. A. Sentef, A. F. Kemper, A. Georges, and C. Kollath, Theory of light-enhanced phonon-mediated superconductivity, Phys. Rev. B 93, 144506 (2016).

[12] M. Knap, M. Babadi, G. Refael, I. Martin, and E. Demler, Dynamical Cooper pairing in nonequilibrium electronphonon systems, Phys. Rev. B 94, 214504 (2016).

[13] L. D'Alessio and M. Rigol, Long-Time Behavior of Isolated Periodically Driven Interacting Lattice Systems, Phys. Rev. X 4, 041048 (2014).

[14] A. Lazarides, A. Das, and R. Moessner, Equilibrium states of generic quantum systems subject to periodic driving, Phys. Rev. E 90, 012110 (2014).

[15] A. Hermann, M. Yuta, E. Martin, and P. Werner, Floquet prethermalization in the resonantly driven Hubbard model, Europhys. Lett. 120, 57001 (2017).

[16] T. Kaneko, T. Shirakawa, S. Sorella, and S. Yunoki, Photoinduced $\eta$ Pairing in the Hubbard Model, Phys. Rev. Lett. 122, 077002 (2019).

[17] D. Fausti, R. I. Tobey, N. Dean, S. Kaiser, A. Dienst, M. C. Hoffmann, S. Pyon, T. Takayama, H. Takagi, and 
A. Cavalleri, Light-induced superconductivity in a stripeordered cuprate, Science 331, 189 (2011).

[18] M. A. Sentef, A. Tokuno, A. Georges, and C. Kollath, Theory of Laser-Controlled Competing Superconducting and Charge Orders, Phys. Rev. Lett. 118, 087002 (2017).

[19] C. N. Yang, Concept of off-diagonal long-range order and the quantum phases of liquid He and of superconductors, Rev. Mod. Phys. 34, 694 (1962).

[20] J. de Boer, V. E. Korepin, and A. Schadschneider, $\eta$ Pairing as a Mechanism of Superconductivity in Models of Strongly Correlated Electrons, Phys. Rev. Lett. 74, 789 (1995).

[21] F. H. L. Essler, V.E. Korepin, and K. Schoutens, New Exactly Solvable Model of Strongly Correlated Electrons Motivated by High- $t_{c}$ Superconductivity, Phys. Rev. Lett. 68, 2960 (1992).

[22] F. H. L. Essler, H. Frahm, F. Göhmann, A. Klümper, and V.E. Korepin, The One-Dimensional Hubbard Model (Cambridge University Press, Cambridge, England, 2005).

[23] C. N. Yang, $\eta$ Pairing and Off-Diagonal Long-Range Order in a Hubbard Model, Phys. Rev. Lett. 63, 2144 (1989).

[24] L. Sewell, Off-diagonal long-range order and the Meissner effect, J. Stat. Phys. 61, 415 (1990).

[25] H. T. Nieh, G. Su, and B.-H. Zhao, Off-diagonal long-range order: Meissner effect and flux quantization, Phys. Rev. B 51, 3760 (1995).

[26] S. Kruchinin, S. Aono, and H. Nagao, Modern Aspects of Superconductivity: Theory of Superconductivity (World Scientific, Singapore, 2010).

[27] P. Monthoux and D. Pines, Spin-Fluctuation-Induced Superconductivity in the Copper Oxides: A Strong Coupling Calculation, Phys. Rev. Lett. 69, 961 (1992).

[28] P. Monthoux, A. V. Balatsky, and D. Pines, Toward a Theory of High-Temperature Superconductivity in the Antiferromagnetically Correlated Cuprate Oxides, Phys. Rev. Lett. 67, 3448 (1991).

[29] U. Schneider, L. Hackermüller, J. P. Ronzheimer, S. Will, S. Braun, T. Best, I. Bloch, E. Demler, S. Mandt, D. Rasch, and A. Rosch, Fermionic transport and out-of-equilibrium dynamics in a homogeneous Hubbard model with ultracold atoms, Nat. Phys. 8, 213 (2012), article.

[30] D. Jaksch and P. Zoller, The cold atom Hubbard toolbox, Ann. Phys. (Amsterdam) 315, 52 (2005).

[31] M. Lewenstein, A. Sanpera, V. Ahufinger, B. Damski, A. Sen De, and U. Sen, Ultracold atomic gases in optical lattices: Mimicking condensed matter physics and beyond, Adv. Phys. 56, 243 (2007).

[32] See Supplemental Material at http://link.aps.org/ supplemental/10.1103/PhysRevLett.123.030603 for (a) numerical details, (b) proof of dephasing induced order in arbitrary systems with multiple symmetries, (c) the robustness of the dephasing protocol to symmetry-breaking dephasing, (d) a proposal for the experimental implementation of the spin-dephased Hubbard model, and (e) further plots, which includes Refs. [33-39].

[33] A. Datta and G. Vidal, Role of entanglement and correlations in mixed-state quantum computation, Phys. Rev. A 75, 042310 (2007).
[34] V. V. Albert, B. Bradlyn, M. Fraas, and L. Jiang, Geometry and Response of Lindbladians, Phys. Rev. X 6, 041031 (2016).

[35] M. Lewenstein, A. Sanpera, and V. Ahufinger, Ultracold atoms in optical lattices: Simulating quantum many-body systems, Contemp. Phys. 54, 112 (2013).

[36] D. D. Awschalom, L. C. Bassett, A. S. Dzurak, E. L. Hu, and J. R. Petta, Quantum spintronics: Engineering and manipulating atom-like spins in semiconductors, Science 339, 1174 (2013).

[37] M. Žnidarič, Relaxation times of dissipative many-body quantum systems, Phys. Rev. E 92, 042143 (2015).

[38] T. Can, Random Lindblad dynamics, arXiv:1902.01442.

[39] O. Penrose and L. Onsager, Bose-Einstein condensation and liquid helium, Phys. Rev. 104, 576 (1956).

[40] A. Klein, M. Bruderer, S. R. Clark, and D. Jaksch, Dynamics, dephasing and clustering of impurity atoms in BoseEinstein condensates, New J. Phys. 9, 411 (2007).

[41] M. Bruderer, A. Klein, S. R. Clark, and D. Jaksch, Polaron physics in optical lattices, Phys. Rev. A 76, 011605(R) (2007).

[42] M. Theis, G. Thalhammer, K. Winkler, M. Hellwig, G. Ruff, R. Grimm, and J.H. Denschlag, Tuning the Scattering Length with an Optically Induced Feshbach Resonance, Phys. Rev. Lett. 93, 123001 (2004).

[43] S. Inouye, M. R. Andrews, J. Stenger, H.-J. Miesner, D. M. Stamper-Kurn, and W. Ketterle, Observation of Feshbach resonances in a Bose-Einstein condensate, Nature (London) 392, 151 (1998).

[44] B. Buca, J. Tindall, and D. Jaksch, Non-stationary coherent quantum many-body dynamics through dissipation, Nat. Commun. 10, 1730 (2019).

[45] A. J. Daley, Quantum trajectories and open many-body quantum systems, Adv. Phys. 63, 77 (2014).

[46] S. R. White, Density Matrix Formulation for Quantum Renormalization Groups, Phys. Rev. Lett. 69, 2863 (1992).

[47] G. Vidal, Efficient Classical Simulation of Slightly Entangled Quantum Computations, Phys. Rev. Lett. 91, 147902 (2003).

[48] S. Al-Assam, S. R. Clark, and D. Jaksch, The tensor network theory library, J. Stat. Mech. (2017) 093102.

[49] E. Ilievski, J. De Nardis, B. Wouters, J.-S. Caux, F. H. L. Essler, and T. Prosen, Complete Generalized Gibbs Ensembles in an Interacting Theory, Phys. Rev. Lett. 115, 157201 (2015).

[50] M. Rigol, V. Dunjko, V. Yurovsky, and M. Olshanii, Relaxation in a Completely Integrable Many-Body Quantum System: An $A b$ Initio Study of the Dynamics of the Highly Excited States of 1D Lattice Hard-Core Bosons, Phys. Rev. Lett. 98, 050405 (2007).

[51] L. Vidmar and M. Rigol, Generalized Gibbs ensemble in integrable lattice models, J. Stat. Mech. (2016) 064007.

[52] J. Mur-Petit, A. Relaño, R. A. Molina, and D. Jaksch, Revealing missing charges with generalised quantum fluctuation relations, Nat. Commun. 9, 2006 (2018).

[53] B. Buča and T. Prosen, A note on symmetry reductions of the Lindblad equation: Transport in constrained open spin chains, New J. Phys. 14, 073007 (2012). 
[54] D. A. Lidar, I. L. Chuang, and K. B. Whaley, DecoherenceFree Subspaces for Quantum Computation, Phys. Rev. Lett. 81, 2594 (1998).

[55] J. E. Hirsch, Attractive Interaction and Pairing in Fermion Systems with Strong On-Site Repulsion, Phys. Rev. Lett. 54, 1317 (1985).
[56] G. Kotliar and J. Liu, Superexchange mechanism and $d$-wave superconductivity, Phys. Rev. B 38, 5142 (1988).

[57] J. Dukelsky, S. Pittel, and G. Sierra, Colloquium: Exactly solvable Richardson-Gaudin models for many-body quantum systems, Rev. Mod. Phys. 76, 643 (2004). 